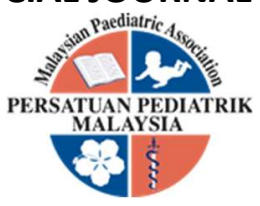

\title{
ACCIDENTAL MAGNET INGESTION IN A TODDLER: A DIAGNOSTIC DILEMMA
}

\author{
Ng Benjamin Wei Liang', Ooi Wei Keong', Prahalad Ramanathan'3 , Toh Teck Hock ${ }^{1,4}$
}

\begin{abstract}
Foreign body ingestion is common in children. Ingestion of foreign bodies with magnetic properties are less common, but can be associated with more severe complication, especially involving multiple magnets. We present a 16-month-old boy with developmental delay who developed small bowel perforation following multiple magnet ingestion. He had three days history of vomiting and abdominal distension associated with pain, suggestive of an acute intestinal obstruction. Abdominal imaging confirmed foreign body ingestion, and an exploratory laparotomy revealed two magnets attracted across the intestinal lining with resultant intestinal obstruction and perforation. Surgical removal of the magnets and the necrotic bowel tissue was complicated by post-operative wound dehiscence. After secondary suturing, the child subsequently made a complete recovery. We discuss the diagnostic dilemma in multiple magnet ingestion along with the importance of early recognition and prompt management due to the risk of more severe gastrointestinal complication associated with delayed intervention.
\end{abstract}

Keywords: Magnet, foreign body, accidental ingestion, bowel obstruction, children

DOI: $10.51407 / \mathrm{mjpch} . v 27 i 1.128$

\section{Introduction}

Foreign body (FB) ingestion is a common problem amongst children under five years of age, especially those with developmental disabilities. [1]. In most cases, FB such as coins, buttons and batteries undergo spontaneous passage without complication [2]. However, some FB like magnets can cause gastrointestinal symptoms such as vomiting and pain for which urgent medical care is required. Magnet ingestion accounts for a small fraction of reported cases of FB ingestion worldwide, but there has been a rising incidence in the past decade [1-3]. Although rare, accidental ingestion of magnets can result in pressure necrosis, intestinal perforation or obstruction, which can be lifethreatening. Differentiating magnets from other less serious FB ingestions can be challenging due to their non-specific radiological findings and clinical presentation. A few authors had previously discussed on the imaging features and modalities to improve the diagnosis of magnet
Received: 09 November 2020; Accepted revised manuscript: 23 February 2021

Published online: 01 June 2021 ingestion but these may not be readily available in resource limited institutions. We describe a case of a 16-month-old boy with developmental delay who presented to a district hospital and later developed bowel obstruction and perforation as a consequence of accidental ingestion of multiple magnets.

\section{Case Presentation}

A 16-month-old boy presented to a district hospital without specialist with three days history of vomiting, progressive abdominal distension and pain. On examination, his growth parameters were appropriate for age with a height of $80 \mathrm{~cm}$ $\left(50^{\text {th }}\right.$ percentile $)$ and weight of $10.6 \mathrm{~kg}\left(50^{\text {th }}\right.$

\footnotetext{
'Department of Paediatrics, Sibu Hospital

${ }^{2}$ Department of Surgery, Sibu Hospital

${ }^{3}$ Department of Diagnostic Imaging, Sibu Hospital

${ }^{4}$ Clinical Research Centre, Sibu Hospital, Sibu, Sarawak, Malaysia.

Corresponding Author:

Dr. Benjamin Ng Wei Liang; Department of Paediatrics, Sibu Hospital, KM8, Jalan Ulu Oya, 96000, Sibu, Sarawak, Malaysia.

Tel: +6084238888 (ext. 7201) Email: benng85@gmail.com
} 
percentile). He had a temperature of $37.5^{\circ} \mathrm{C}$ with a soft, non-tender but mildly distended abdomen. He was initially managed conservatively as acute gastroenteritis and admitted for observation with maintenance intravenous fluids. The parents were uncertain if the child had accidentally ingested any FB, but they reported that the child had a habit of placing objects in his mouth. There was no remarkable past medical or surgical history. Incidentally, the child was also found to have poor eye contact and pre-verbal skills. He spoke in jargons with no meaningful words, but his motor milestones appeared to be normal for his age. Developmentally, he was delayed with a mental age of about one-year-old with features suspicious of autism.

On day two of admission, the child became irritable and lethargic. His perfusion was good but he was tachycardic with a heart rate of 130 beats/min. His abdomen became more distended with mild tenderness and bowel sounds were sluggish. A nasogastric tube inserted drained a large amount of bilious fluid.

Urgent abdominal x-ray showed dilated small bowel loops and paucity of distal bowel gas. There was no evidence of pneumoperitoneum. There was a radio-opaque FB of metallic density, resembling a singular button cell at the level of the fourth lumbar vertebra [Figure 1 \& Figure 2].

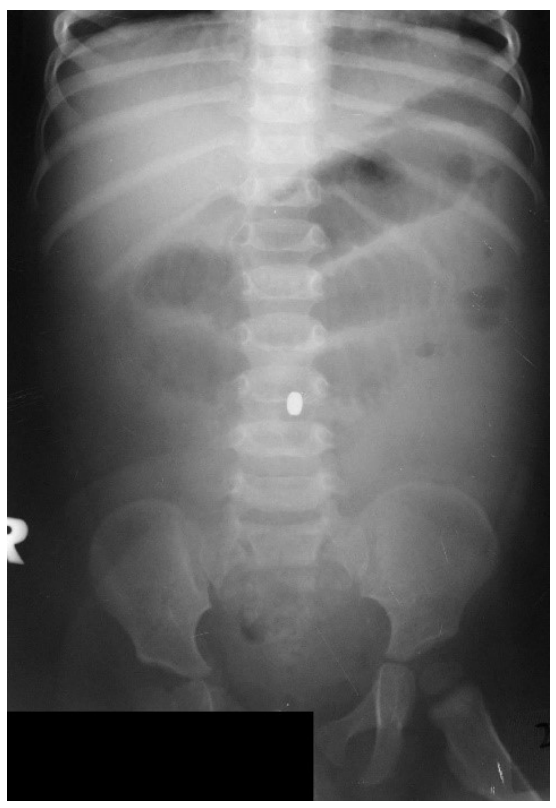

Figure 1. Anterior-posterior view abdominal radiograph showing a radio-opaque foreign body of metallic density at the fourth lumbar vertebra level.

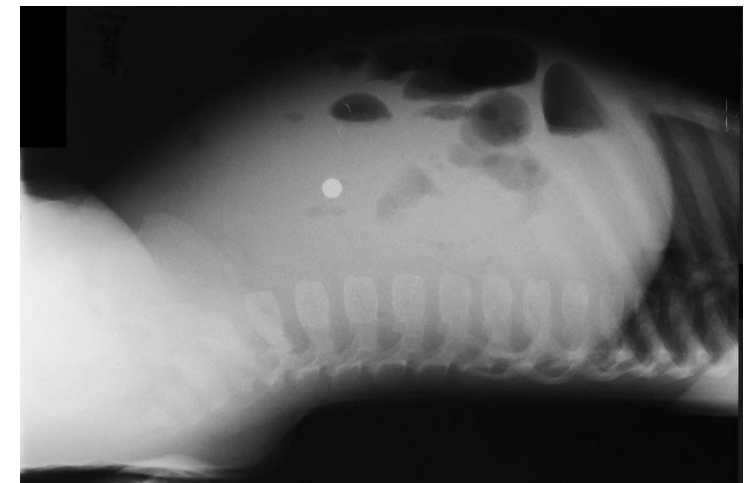

Figure 2. Lateral view radiograph shows dilated bowel loops and paucity of the distal bowel gas with no pneumoperitoneum and the foreign body.

After consulting a surgeon from a tertiary centre, a provisional diagnosis of FB ingestion with bowel obstruction was made. The child was given intravenous fluid bolus of normal saline followed by a dose of osmotic laxative, and then observed for spontaneous passage of the FB, as expected of a button cell. A repeated abdominal x-ray after five hours showed a non-progressing FB and persistent small bowel dilatation [Figure 3].

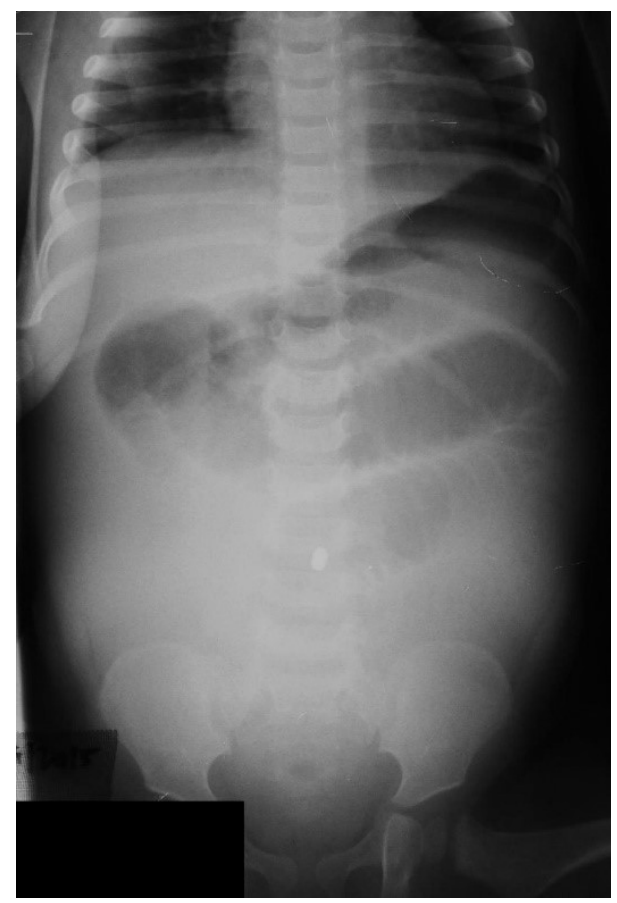

Figure 3. Abdominal radiograph repeated five hours later shows persistently dilated bowels with static foreign body.

His condition deteriorated further and was transferred to the tertiary centre on the same day for further management. Intravenous 
cefoperazone $(50 \mathrm{mg} / \mathrm{kg})$ and metronidazole $(7.5 \mathrm{mg} / \mathrm{kg})$ were commenced. An urgent abdominal ultrasound showed small bowel dilatation with inter-loop fluid and minimal free fluid in the abdomen. His abdomen gradually became tenser with generalised tenderness. He had reduced urine output and increasing tachycardia, requiring more fluid resuscitation. As there was no clinical improvement and resolution of symptoms, instead, the child deteriorated, an exploratory laparotomy was arranged. Intraoperative findings showed an accumulation of non-foul smelling ascitic fluid within the peritoneum. The small bowel had perforation at two different sites; one at the proximal small bowel and one distally. There were two small magnets at the site of the perforation. They were attracted across the lining of the intestine, creating a loop with resultant obstruction [Figure 4]. Besides, there was moderate amount of pus localised at the perforation site. The necrotic tissue at the perforation site was excised and primary repair of the bowel perforation with $\operatorname{PDS}^{\circ} 3 / 0$ was done.

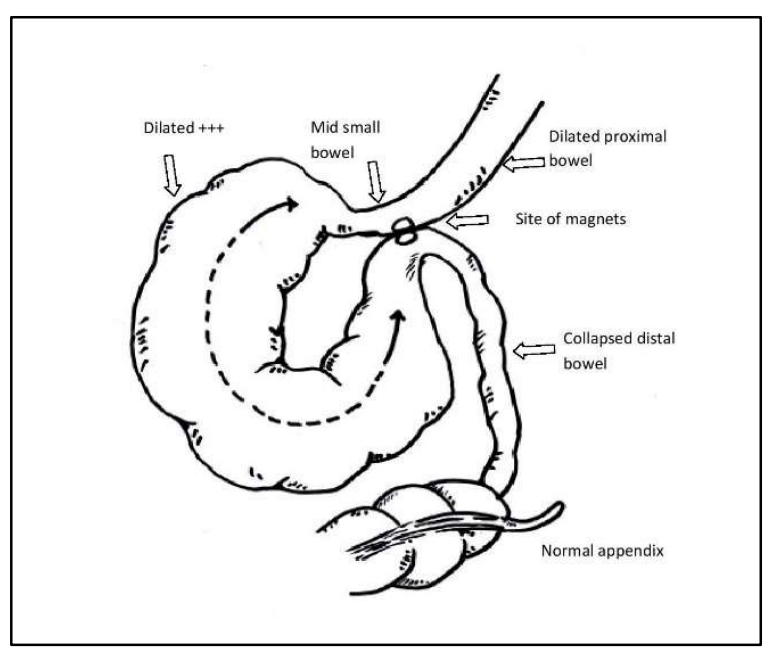

Figure 4. Illustration showing two small magnets attracted across the bowel lining at two different points creating a loop with resultant obstruction, and later perforation.

His parents later reported that the magnets removed intra-operatively resembled those from his alphabetical refrigerator magnets which he had been playing with at home. Post operatively there was wound breakdown, and the child was given a new course of oral cefuroxime $(15 \mathrm{mg} / \mathrm{kg})$ and daily wound dressing. Secondary suturing was done at day 9 post surgery and he made a complete recovery by day 16 . The child was given a follow up clinic at a local child development unit and a diagnosis of autism spectrum disorder was made eventually.

\section{Discussion}

We presented a case of a toddler with an undiagnosed developmental disorder who had a bowel obstruction and perforation as a result of accidental magnet ingestion. Magnet ingestion without a clear history is difficult to differentiate from other non-serious FB ingestion, and its nonspecific clinical features have caused delay in the diagnosis and surgical intervention.

Foreign body ingestion is a rare cause of bowel obstruction as almost $90 \%$ of the ingested objects pass through the gastrointestinal tract spontaneously without complication [2]. Often, a conservative approach of close monitoring and serial imaging is recommended for $\mathrm{FB}$ ingestion. Magnetic FB ingestion, on the other hand, can be potentially life-threatening especially among children, with gastrointestinal complications requiring surgical intervention reported in up to $75 \%$ of cases [3]. Yet, these potential hazards are now easily accessible to young children as the reduced costs and improved manufacturing technology had allowed high-powered magnets to expand its use in many consumer products, including children's toys.

Like most other $\mathrm{FB}$, ingestion of a single magnet can be managed conservatively [3]. However, when two or more magnets are ingested, they can attract each other across the bowel walls resulting in bowel obstruction, perforation and fistula formation, as demonstrated in our case. Therefore, a more active and earlier surgical approach is warranted if multiple magnets are ingested as they have a higher incidence of gastrointestinal complications [4]. The key here, therefore, is first to determine if a single or multiple magnets were being ingested. Unfortunately, as in our case, this is incredibly difficult most of the time in children.

The diagnosis and management of magnet ingestion in a child are challenging. The clinical signs and symptoms of magnet ingestion are non-specific, often resembling those of other more common gastrointestinal disorders, leading to delay in diagnosis. Prompt diagnosis is further complicated as FB ingestion is typically unwitnessed and accurate history of the nature of 
the FB is hard to obtain from children, even more so in a child with developmental concerns. Children with developmental delays such as autism, attention deficit hyperactivity disorder and learning disability are associated with a higher risk of $\mathrm{FB}$ ingestion due to their tendency to put objects into their mouths $[1,5]$.

Radiographic examination is an easily accessible modality and may aid in the diagnosis. Unfortunately, they lack the sensitivity to ascertain if the FB is magnetic or to identify between single and multiple magnet ingestion. Multiple magnets may adhere to each other densely and may appear as a singular object on radiographs, without gaps or notches between them $[5,6]$. Magnifying the FB on the radiograph may help to better appreciate the notches and gaps but this would require computerised images which is not readily available in our setting. Computed tomography (CT) scan is reported to be helpful in cases of unwitnessed ingestion without an appropriate history and when radiographs are not readily available. However, FB with metallic density can create artefact on CT images that can prevent an accurate identification of the object or the quantity of $\mathrm{FB}$ ingested [7]. In our case, the two magnets were stacked together across the intestinal lining, forming a closed loop and misleadingly resembled a single button cell in the bowel lumen on the serial radiographs from different views. CT imaging may not be able to clearly distinguish the FB or provide additional information to the management of our patient.

Lee et al [2] reported a case of a child with multiple magnet ingestion who was initially managed as enteritis and serial radiographs showed static FB of metallic density similar to that in our case which was suggestive of failure to progress through the bowel. Findings of bowel obstruction coupled with a non-progressing FB on serial images should raise suspicion of multiple magnetic elements with entrapped bowels which can lead to numerous other gastrointestinal complications. Earlier surgical intervention might potentially lessen the severity of the complication in our case, if possibility of magnet ingestion was considered from the initial encounter.

This case serves to illustrate the diagnostic dilemma in dealing with $\mathrm{FB}$ with magnetic properties. While serial radiograph is the recommended approach for most FB ingestions, one should be aware of the limitation of radiographs in accurately determining the nature of the FB, particularly in those cases of unwitnessed ingestion and in young pre-verbal children. Additionally, multiple magnet ingestion should be considered in a non-progressing radioopaque FB with metallic density seen on serial radiographs. The case also reminds us as clinicians to always advocate parents on the introduction of age-appropriate toys to children and to be vigilant to the potential hazards in the child's environment. Anticipatory guidance for injury prevention to parents with young children is a vital part of child health care.

In summary, there is still a lack of awareness of the potential complications that can arise from multiple magnet ingestion among caretakers and medical personnel. Clinicians should regard all FB ingestion with a high index of suspicion to rule out multiple magnet ingestion in cases of nonprogressing $\mathrm{FB}$ on serial imaging and surgical intervention should be expedited at the very first sign of gastrointestinal complication.

\section{Acknowledgment}

We thank Director General of Health Malaysia for his permission to publish this case report. We also thank the parents for providing us the written consent to presenting the information and images in this paper.

\section{References}

[1] George AT, Motiwale S. Magnets, children and the bowel: a dangerous attraction? World J Gastroentrol 2012 October 14; 18(38):53248.

[2] Lee BK, Ryu HH, Moon JM, Jeung KW. Bowel perforation induced by multiple magnet ingestion. Emerg Med Australas 2010; 22:18991.

[3] Hussain SZ, Bousvaros A, Gilger $M$, et al. Management of ingested magnets in children. J Pediatr Gastroenterol Nutr 2012; 55:239-42.

[4] Adikibi BT, Amold $M$, van Niekerk G, Alexander A, Numanoglu A, Millar AJW. 
Magnetic bead toy ingestion: uses and disuses in children. Pediatr Surg Int 2013; 29:741-744.

[5] Centers for Disease Control and Prevention. Gastrointestinal injuries from magnet ingestion in children-United States, 20032006. MMWR 2006; 55:1296-300.

[6] Butterworth J, Feltis B. Toy magnet ingestion in children: revising the algorithm. $J$ Pediatr Surg 2007; 42:e3-5.

[7] Evangelos Blevrakis, Maria Raissaki, Sofia Xenaki, Elissavet Astyrakaki, Nelli Kholcheva, and Emmanuel Chrysos; Multiple magnet ingestion causing intestinal obstruction and entero-enteric fistula: Which imaging modality besides radiographs? A case report; Ann Med Surg (Lond); 2018 Jul; 31: 29-33. 